\title{
Formal Sensitivity Computation of Magnetic Moment Method
}

\author{
H. L. Rakotoarison ${ }^{1}$, V. Ardon ${ }^{2}$, O. Chadebec ${ }^{1}$, B. Delinchant ${ }^{1}$, S. Guerin ${ }^{1}$, and J.-L. Coulomb ${ }^{1}$ \\ ${ }^{1}$ Grenoble Electrical Engineering Laboratory (G2ELab), CNRS-UJF/INPG, 38402 Saint Martin d'Héres, France \\ ${ }^{2}$ Schneider Electric, Grenoble 38000, France
}

\begin{abstract}
This paper presents a methodology for computing formally sensitivities of the magnetic moment method. This numerical method is known to be very accurate and light to compute force and torque acting on an open field problem. We propose an approach which computes formal derivatives with respect to the geometrical parameters of the device. Once the sensitivities are obtained, gradient based optimization algorithm can be used.
\end{abstract}

Index Terms-Magnetostatic, moment methods, optimization, sensitivity.

\section{INTRODUCTION}

$\mathbf{T}$ HE MODELING of devices containing ferromagnetic material (iron, nickel, cobalt, and their alloys. etc.) in magnetostatic is achieved thank to numerical approaches like finite element method (FEM), boundary element method (BEM), method of moments (MoM). In a design process, the modeling must be coupled with an optimization process. Even if stochastic method (like genetic algorithm) can be applied, these techniques lead to a very important computation time increasing drastically with the number of parameters. The use of a surface response can partially solve this problem but the number of parameters is still limited. Gradient based methods (like Quasi-Newton algorithm) are very efficient solution. It is then necessary to compute the sensitivity of the objective function versus the parameters. Numerical sensitivities calculation using finite difference is fairly straightforward and an easy way to achieve it, but it is not a robust technique. Hence, we present an original method based on formal differentiation of the MoM approach.

\section{Method OF Moments}

The MoM is an accurate and light numerical approach dedicated to the modeling of electromagnetic devices. In this method, only active regions (i.e., ferromagnetic parts of the device) are meshed. Thus it is particularly well adapted for the modeling of simple radiated devices with few ferromagnetic parts in comparison of the total air region.

The MoM is based on the discretization of the system (1), associated with a point matching approach applied at the barycenter of each elements [1]

$$
\left.\mathbf{H}(P)=\mathbf{H}_{\mathrm{ext}}(P)+\frac{1}{4 \pi} \iint_{V} \int\left(\frac{3(\mathbf{M} . \mathbf{r})}{\mathbf{r}^{5}} r-\frac{1}{\mathbf{r}^{3}} \mathbf{M}\right) d v\right) .
$$

By combining (1) with the magnetic law, we get the following system of equations:

$$
\left\{\begin{array}{l}
\mathbf{H}=\mathbf{H}_{\mathrm{ext}}+\mathbf{A} \cdot \mathbf{M} \\
\mathbf{H}=\mathbf{f}(\mathbf{M})
\end{array}\right.
$$

Digital Object Identifier 10.1109/TMAG.2007.915294

Color versions of one or more of the figures in this paper are available online at http://ieeexplore.ieee.org.

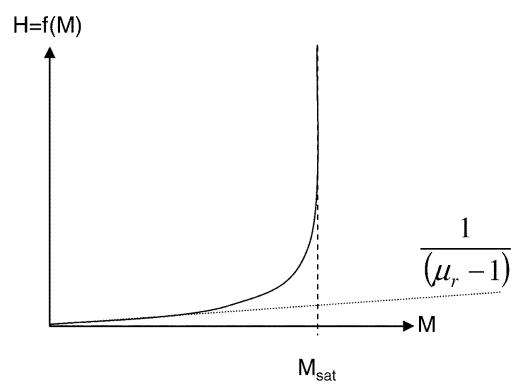

Fig. 1. Reversed magnetization law of a ferromagnetic material, the function is characterized by its slope and the saturation magnetization.

$H$ and $H_{\text {ext }}$ are respectively the magnetic field and the applied external field generated by the sources (conductors, permanent magnet, etc. ) at the barycenter of the element; $A$ is the interaction matrix between elements. It depends on the geometrical parameters of the system and mesh processes, $M$ is the magnetization of the material and $f(M)$ a function which depends on the material properties. The magnetization law of a ferromagnetic material is shown in the Fig. 1; this function is characterized by its slope at the origin and its saturation value.

If the material has a linear characteristic (paramagnetic, diamagnetic material, and ferromagnetic material working at low excitation field) the reversed magnetization law function becomes simpler

$$
f(M)=\frac{1}{\left(\mu_{r}-1\right)} M
$$

where $\mu_{r}$ is the relative permeability of the material. For nonlinear behavior of a ferromagnetic material, the reversed magnetization law is given by

$$
f(M)=\frac{M_{\mathrm{sat}}}{\frac{\pi}{2} \cdot\left(\mu_{r}-1\right)} \cdot \operatorname{tg}\left(\frac{\pi}{2} \frac{M}{M_{\mathrm{sat}}}\right) .
$$

Reducing the system (2) into one set of nonlinear equations leads to

$$
\mathbf{R}(\mathbf{M})=\mathbf{H}_{\mathrm{ext}}+\mathbf{A} \cdot \mathbf{M}-\mathbf{f}(\mathbf{M})=0
$$

In fact, (5) has a vector form. So, the scalar function $f(M)$ needs transformation (6) to be a vectorial function

$$
\mathbf{f}(\mathbf{M})=f(\|\mathbf{M}\|) \frac{\mathbf{M}}{\|\mathbf{M}\|} \text {. }
$$




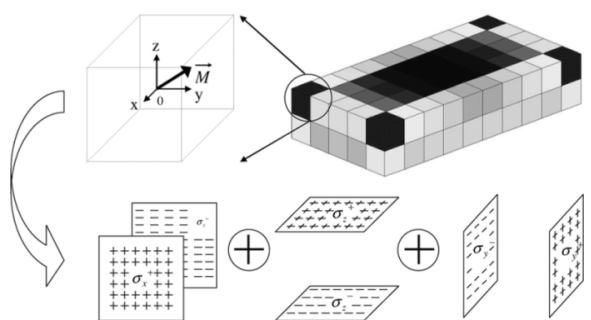

Fig. 2. Surface charge approach, used to compute force and torque calculation after solving the magnetization state of the system.

The ferromagnetic volume is meshed into $N$ elements; magnetization is supposed to be uniform on each of them. Equation (5) is a system of $3 \times \mathrm{N}$ equations ( 3 as the $\mathrm{x}, \mathrm{y}$, and $\mathrm{z}$ coordinate, an $\mathrm{N}$ as the number of element). The unknown variable is the magnetization $\mathrm{M}$, which is a vector with the same size as the system (5).

\section{A. Solving the Magnetization State of the System}

Classically, it remains to solve (5) to get the magnetizations. For a linear material, plugging (4) into (5) gives a simple linear system

$$
\left[\frac{\mathbf{I}}{\left(\mu_{r}-1\right)}-\mathbf{A}\right] \cdot \mathbf{M}=\mathbf{H}_{\mathrm{ext}} .
$$

In the other hand, for nonlinear material the relation (5) is solved by using Newton-Raphson algorithm. At the end of the resolution process, magnetization of each element is obtained.

\section{B. Computing Forces and Torques Acting on the Body}

Once the magnetization state of the system is solved, forces and torques acting on the devices can be computed by using the well-known equivalent magnetic charge approach. The charge distribution is expressed by

$$
\begin{aligned}
\sigma_{\mathrm{v}} & =-\operatorname{div}(\mathbf{M}) \\
\sigma_{s} & =\mathbf{M} . \mathbf{n}
\end{aligned}
$$

where $n$ is the external normal of the surface. As the magnetization $\mathbf{M}$ is supposed to be uniform inside an element, volume density charge $\sigma_{v}=0$, and the surface density charge $\sigma_{\mathrm{s}}$ can be evaluated by projecting the magnetization vector on the six surfaces of the element (Fig. 2). If $\mu_{0}, \sigma_{s_{i j}}, \mathbf{P}_{s_{i j}}$ are respectively the permeability of the vacuum, the charge density of the surface $j$ owned by the element $i$ and the point which describes the surface of the element.

The force calculation applied on the $\mathrm{N}$ elements of the segmented bloc is given by (9).

$$
\mathbf{F}=\mu_{0} \sum_{i=1}^{N} \sum_{j=1}^{6} \iint_{s_{i j}} \sigma_{s_{i j}} . \mathbf{H}_{\mathrm{ext}}\left(\mathbf{P}_{s_{i j}}\right) . d s
$$

From (9), the computation of the torque is straightforward

$$
\boldsymbol{\Gamma}=\mu_{0} \sum_{i=1}^{N} \sum_{j=1}^{6} \iint_{s_{i j}} \mathbf{O P}_{s_{i j}}\left[\sigma_{s_{i j}} \cdot \mathbf{H}_{\mathrm{ext}}\left(\mathbf{P}_{s_{i j}}\right)\right] . d s
$$

where $\mathrm{O}$ is the pivot point where the torque is computed.

Let us note that in (5), the points where the vectors of external field $\mathrm{H}_{\mathrm{ext}}$ are computed in the barycenter of each element, whereas those of (9) and (10), are computed on each surface of each element.

From here, let us consider a function $\Phi$ which represents, the torque or the force applied on the ferromagnetic material or its radiated magnetic field at an observation point

$$
\Phi=\Phi\left(\mathbf{M}, \mathbf{H}_{\mathrm{ext}}, p\right) .
$$

$\Phi$ depends on the resolved magnetization $M$, on the applied external field $H_{\text {ext }}$, and on the parameter $p$ of the model.

\section{Formal Derivation of the Method}

\section{A. Drawbacks of the Finite Difference Approach}

Finite difference of a function $\Phi$ versus a parameter $p$ is computed easily by using

$$
\frac{d \Phi}{d p}=\frac{\Phi(p+h)-\Phi(p)}{h} .
$$

The advantage of this method is the easiness in its implementation, but users are aware of its well-known problem, it occurs when the user supplies the right step "h." Because, its choice depends tightly of the variation of the function (which could not be evaluated), and the accuracy of the computer which implement the numerical code.

This approach presents another significant weakness because the function $\Phi$ needs to be evaluated two times. This can be time consuming if this function calls an iterative algorithm, especially in our case the nonlinear implicit solver, moreover if derivative versus $n$ parameter is required, the algorithm will be called $n+1$ times.

The formal approach allows solving those problems, because it involves only one call to the function even if we need the evaluation of the partial derivative of the function versus the $n$ parameters of the model.

\section{B. Classical Approach}

The differential of the function $\Phi$ is given by

$$
\begin{aligned}
& d \Phi=\left.\frac{\partial \Phi}{\partial \mathbf{M}}\right|_{H_{\mathrm{ext}}, p} \cdot d \mathbf{M}+\left.\frac{\partial \Phi}{\partial \mathbf{H}_{\mathrm{ext}}}\right|_{M, p} \\
& \cdot d \mathbf{H}_{\mathrm{ext}}+\left.\frac{\partial \Phi}{\partial p}\right|_{M, H_{\mathrm{ext}}} \cdot d p .
\end{aligned}
$$

So, the classical computation of the sensitivity of $\Phi$ versus $p$ can be expressed

$$
\frac{d \Phi}{d p}=\left.\frac{\partial \Phi}{\partial \mathbf{M}}\right|_{H_{\mathrm{ext}}, p} \frac{d \mathbf{M}}{d p}+\left.\frac{\partial \Phi}{\partial \mathbf{H}_{\mathrm{ext}}}\right|_{M, p} \frac{d \mathbf{H}_{\mathrm{ext}}}{d p}+\left.\frac{\partial \Phi}{\partial p}\right|_{M, H_{\mathrm{ext}}} .
$$

The evaluation of

$$
\left.\frac{\partial \Phi}{\partial \mathbf{M}}\right|_{H_{\mathrm{ext}}, p},\left.\left.\frac{\partial \Phi}{\partial \mathbf{H}_{\mathrm{ext}}}\right|_{M, p} \quad \frac{\partial \Phi}{\partial p}\right|_{M, H_{\mathrm{ext}}}
$$

depends on how $\Phi$ is expressed, and depend strongly on the mesh method.

$\left(d \mathbf{H}_{\text {ext }} / d p\right)$ can be easily computed by using formal derivation on the equations modeling the magnetic field produced by source (permanent magnet and conductors) [3]. 
$(d \mathbf{M} / d p)$ is obtained such that we have the following: the derivation of (5) according to the parameter $p$ gives

$$
\begin{aligned}
\frac{d \mathbf{H}_{\text {ext }}}{d p}+\frac{\mathbf{A} \cdot d \mathbf{M}}{d p} & \frac{d \mathbf{A}}{d p} \cdot \mathbf{M} \\
& -\left[\left.\frac{\partial f(\mathbf{M})}{\partial M}\right|_{p} \cdot \frac{d \mathbf{M}}{d p}+\left.\frac{\partial f(\mathbf{M})}{\partial p}\right|_{M}\right]_{0} .
\end{aligned}
$$

Then, the arrangement of (15) gives a linear system like (15), which can be solved easily

$$
\left[\mathbf{A}-\left.\frac{\partial f(\mathbf{M})}{\partial M}\right|_{p}\right] \cdot \frac{d \mathbf{M}}{d p}=\left.\frac{\partial f(\mathbf{M})}{\partial p}\right|_{M}-\frac{d \mathbf{H}_{\text {ext }}}{d p}-\frac{d \mathbf{A}}{d p} \cdot \mathbf{M} .
$$

The above equation needs

$$
\begin{aligned}
& \left.\frac{\partial f(\mathbf{M})}{\partial M}\right|_{p}=\frac{\|\mathbf{H}\|}{\|\mathbf{M}\|}+\frac{1}{\|\mathbf{M}\|^{2}}\left[\left.\frac{\partial f(\|\mathbf{M}\|)}{\partial M}\right|_{p}-\frac{\|\mathbf{H}\|}{\|\mathbf{M}\|}\right] \\
& \text { - } \mathbf{M} \cdot \mathbf{M}^{t} \text {. }
\end{aligned}
$$

Then, the sensitivity of $\Phi$ versus p is obtained by combining (13) and the solution of the linear system (16).

Once again, the evaluation of $\left(d \mathbf{H}_{\text {ext }} / d p\right)$ could be expressed formally by applying formal derivation on the model of external field given by permanents magnets or conductors carrying current density as in [3]. The formal computation of $(d \mathbf{A} / d p)$ is possible if the mesh process could be parameterized according the dimension of the ferromagnetic part.

To achieve the jacobian computation (i.e., vector of partial derivative of $\Phi$ versus the $k$ parameters $p$ ), the linear system (16) has to be solved $k$ times or matrix inversion operation is used; thus it can be time consuming in the case of optimization process. A well-known method to overpass this problem is the use of the adjoint state as in [2].

\section{Enhanced Approach by Using the Adjoint State}

Now, let us introduce the adjoint state $\lambda$, which has the same dimension as $M$, and satisfies (18)

$$
\left[\mathbf{A}-\left.\frac{\partial f(\mathbf{M})}{\partial M}\right|_{p}\right]^{\mathbf{t}} \cdot \boldsymbol{\lambda}=\left(\left.\frac{\partial \Phi}{\partial \mathbf{M}}\right|_{p}\right)^{t} .
$$

The transposition of the (18) gives

$$
\boldsymbol{\lambda}^{\mathbf{t}} \cdot\left[\mathbf{A}-\left.\frac{\partial f(\mathbf{M})}{\partial M}\right|_{p}\right]=\left.\frac{\partial \Phi}{\partial \mathbf{M}}\right|_{p} .
$$

Multiplying the (16) by $\lambda^{\mathrm{t}}$ gives

$$
\begin{aligned}
& \lambda^{\mathbf{t}}\left[\mathbf{A}-\left.\frac{\partial f(\mathbf{M})}{\partial M}\right|_{p}\right] \cdot \frac{d \mathbf{M}}{d p} \\
&=\lambda^{\mathbf{t}}\left(\left.\frac{\partial f(\mathbf{M})}{\partial p}\right|_{M}-\frac{d \mathbf{H}_{\mathrm{ext}}}{d p}-\frac{d \mathbf{A}}{d p} \cdot \mathbf{M}\right) .
\end{aligned}
$$

TABLE I

Partial Derivative of Some Term According TO THE TYPE OF THE PARAMETER

\begin{tabular}{ccccc}
\hline \hline $\mathrm{p}$ & $\frac{\partial f(\mathbf{M})}{\partial p}$ & $\frac{d \mathbf{H}_{\text {ext }}}{d p}$ & $\frac{d \mathbf{A}}{d p}$ & $\left.\frac{\partial \Phi}{\partial p}\right|_{M, H_{e x t}}$ \\
\hline $\mathrm{p}_{\alpha}$ & 0 & $\mathbf{x}$ & $\mathbf{x}$ & $\mathbf{x}$ \\
$\mathrm{p}_{\beta}$ & $\mathbf{x}$ & 0 & 0 & 0 \\
$\mathrm{p}_{\gamma}$ & 0 & $\mathbf{x}$ & 0 & $\mathbf{x}$ \\
\hline \hline
\end{tabular}

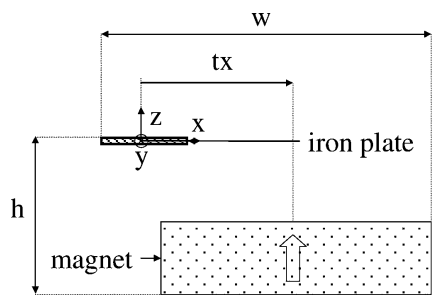

Fig. 3. Irron plate actuated by a permanent magnet. The position of the permanent magnet is characterized by the parameter "tx," and the width and the height of the device is characterized respectively by "w" and "h."

Using the combination of (19) and (20) in (14) gives the finale result shown in

$$
\begin{array}{r}
\frac{d \Phi}{d p}=\boldsymbol{\lambda}^{\mathbf{t}} \cdot\left(\left.\frac{\partial f(\mathbf{M})}{\partial p}\right|_{M}-\frac{d \mathbf{H}_{\text {ext }}}{d p}-\frac{d \mathbf{A}}{d p} \cdot \mathbf{M}\right) \\
\quad+\left.\frac{\partial \Phi}{\partial \mathbf{H}_{\mathrm{ext}}}\right|_{M, p} \cdot \frac{d \mathbf{H}_{\mathrm{ext}}}{d p}+\left.\frac{\partial \Phi}{\partial p}\right|_{M, H_{\mathrm{ext}}}
\end{array} .
$$

So, only one linear system (18) resolution per function $\Phi$ is required to its derivative versus $k$ parameters. This approach is more efficient than (16) if the number of function $\Phi$ is smaller than the number of parameter $p$, which is often the case.

The parameter $p$ can be one of the following kinds.

$\mathrm{p}=\mathrm{p}_{\alpha}$, if $p$ is a geometrical parameter of the ferromagnetic body.

$\mathrm{p}=\mathrm{p}_{\beta}$, if $p$ is a physical parameters of the ferromagnetic material $\left(\mathrm{M}_{\mathrm{sat}}\right.$ and $\left.\mu_{\mathrm{r}}\right)$.

$\mathrm{p}=\mathrm{p}_{\gamma}$, if $p$ acts on the external field (the geometrical or physical parameter of the magnetic field source object like permanent magnet or coil).

$\mathrm{p}=\mathrm{p}_{\delta}$, if $p$ is a mesh parameter. The derivative according this last kind of parameter could not be computed formally because it is a discrete parameter and it does not contribute to the real behavior of the model. Table I gives a guide which shows that some terms in (21) equals to 0 ; crosses mean that the partial derivative exists and needs to be evaluated.

\section{APPLICATION}

Let us study an iron plate actuated by a magnetic field produced by a parallel piped permanent magnet (Fig. 3); it represents a MEMS actuator like [4]. The plate is fixed into a torsion beam and can rotate around the $y$ axis. The magnet is magnetized according the $z$ direction, it has only one degree of freedom which is its translation according the $x$ direction, its position is parameterized by "tx." 


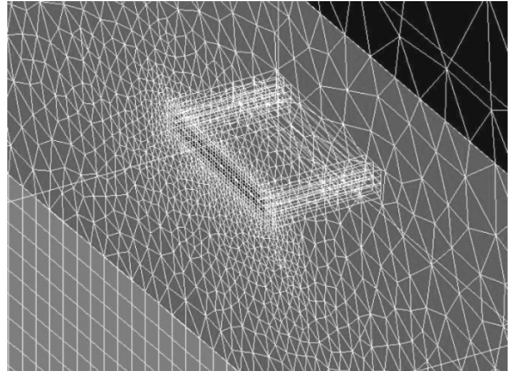

Fig. 4. Device is meshed and simulated on an FEM software, remark the need of the mesh density surrounding the plate.

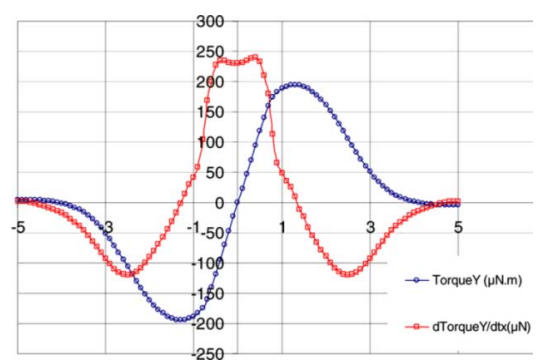

Fig. 5. Behavior of the torque and its formal sensitivity versus the magnet translation, the plate dimensions and orientation remains fixed.

We had meshed and simulated the device by an FEM software (Fig. 4), because of the high ratio between the dimension of the magnet $\left(10 \times 5 \times 5 \mathrm{~mm}^{3}\right)$ and the plate $\left(1 \times 1 \times 0.01 \mathrm{~mm}^{3}\right)$, we have surrounded this last by three level of boxes, the inner box which is closest of the plate is meshed finely and the outer box is meshed coarsely. Finally, the finite element model contains more than 40000 elements. Despite this mesh strategy, the problem is hard to convergence for some particular positions of the magnet. This is one of the reasons which lead us to use the MoM model.

Fig. 5 shows the torque behavior applied on the plate and its formal sensitivity calculation versus the $t x$ parameter. The angular position of the plate is fixed.

\section{OPTIMIZATION}

The derivative is validated; the next step consists to optimize the device; the following are the corresponding specifications:

- Objective function:

- minimize the volume of the device

- Inputs constrains

— physical and geometrical parameters of the iron plate is fixed

— two fixed angles $\left.(\alpha) 1=-20^{\circ}, \alpha 2=15^{\circ}\right)$ for the iron plate rotation.

- Output constrains:

$-\Gamma \min \leqslant \Gamma(\mathrm{t} \times 1, \alpha 1)$.

$-0 \leqslant \Gamma(\mathrm{t} \times 2, \alpha 2)$.

- the difference $(\mathrm{t} \times 2-\mathrm{t} \times 1)$ is fixed.

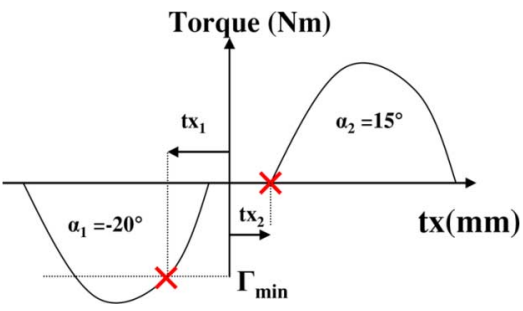

Fig. 6. Specifications applied on the torque behavior.

Fig. 6 helps us to formulate the specifications of the torques. We had adopted an innovative optimization strategy to guarantee the global optimum and an accurate solution of the optimization, it consists in optimizing the model into two steps.

- First, we have chosen randomly inside the space of input constrains, $\mathrm{N}$ initial inputs points. Starting form these points, we have optimized $\mathrm{N}$ times a coarse model of the iron plat (in our case, a subdivision of four elements is enough) by an SQP algorithm. Each optimization spends much iteration but because of the lightness of the coarse model, each process converges quickly into a local optimum; finally we choose the best local optimum, which becomes the global optimum of the coarse solution.

- Then, we had optimized a heavy and accurate model (composed by 100 elements) by supplying as the initial point of the SQP algorithm the corresponding input parameter the global optimum of the coarse solution; this leads to a fast convergence of the heavy model and guarantees a accurate and global optimum.

\section{CONCLUSION}

The approach shows the feasibility of the formal sensitivity calculation applied on the Magnetic Moment Method. The formal sensitivity computation is more robust than the finite difference method for an optimization process based on a gradient algorithm. Finally, we supply an innovative optimization strategy as application of the approach.

\section{REFERENCES}

[1] O. Chadebec, J. L. Coulomb, and F. Janet, IEEE Trans. Magn., vol. 42 , pp. 515-520, 2006

[2] S. Gitosusastro, J. L. Coulomb, and J. C. Sabonnadiere, IEEE Trans. Magn., vol. 25, pp. 2834-2839, 1989.

[3] H. L. Rakotoarison, B. Delinchant, and O. Cugat, in Proc. IEEE CEFC, 2006, p. 441.

[4] J. W. Judy and R. S. Muller, J. Microelectromech. Syst., vol. 6, pp. 249-256, Sep. 1997.

Manuscript received June 24, 2007. Corresponding author: H. L. Rakotoarison (e-mail: lalao.rakotoarison-harijaona@g2elab.inpg.fr). 